\title{
Efficacy and Safety of 12-week Interferon-based Danoprevir Regimen in Patients with Genotype 1 Chronic Hepatitis C
}

\author{
Lai Wei*1, Jia Shang ${ }^{2}$, Yuanji $\mathrm{Ma}^{3}$, Xiaoyuan $\mathrm{Xu}^{4}$, Yan Huang ${ }^{5}$, Yujuan Guan ${ }^{6}$, \\ Zhongping Duan ${ }^{\prime}$, Wenhong Zhang ${ }^{8}$, Zhiliang Gao ${ }^{9}$, Mingxiang Zhang ${ }^{10}$, Jun $\mathrm{Li}^{11}$, Jidong Jia ${ }^{12}$, \\ Yongfeng Yang ${ }^{13}$, Xiaofeng Wen ${ }^{14}$, Maorong Wang ${ }^{15}$, Zhansheng Jia ${ }^{16}$, Bo Ning ${ }^{17}$, \\ Yongping Chen ${ }^{18}$, Yue $\mathrm{Qi}^{19}$, Jie Du ${ }^{20}$, Jianning Jiang ${ }^{21}$, Lixin Tong ${ }^{22}$, Yao Xie ${ }^{23}$ and Jinzi J. Wu ${ }^{24}$ \\ ${ }^{1}$ Beijing Key Laboratory of Hepatitis C and Immunotherapy for Liver Diseases, Beijing, China; ${ }^{2}$ People's Hospital of Henan \\ Province, Henan, China; ${ }^{3}$ West China Hospital, Sichuan University, Chengdu, Sichuan, China; ${ }^{4}$ Peking University People's \\ Hospital, Beijing, China; ${ }^{5}$ Xiangya Hospital of Central South University, Changsha, Hunan, China; ${ }^{6}$ Guangzhou Eighth People's \\ Hospital, Guangzhou, Guangdong, China; 7 Artificial Liver Center, Beijing YouAn Hospital, Capital Medical University, Beijing, \\ China; ${ }^{8}$ Huashan Hospital, Fudan University, Shanghai, China; ${ }^{9}$ The Third Affiliated Hospital of Sun Yat-sen University, \\ Guangzhou, Guangdong, China; ${ }^{10}$ The Sixth People's Hospital of Shenyang, Shenyang, Liaoning, China; ${ }^{11}$ People's Hospital of \\ Jiangsu Province, Nanjing, Jiangsu, China; ${ }^{12}$ Beijing Friendship Hospital, Capital Medical University, Beijing, China; ${ }^{13}$ Nanjing \\ Medical University Affiliated Second Hospital, Nanjing, Jiangsu, China; ${ }^{14}$ Liuzhou People's Hospital, Liuzhou, China; ${ }^{15}$ Liver \\ Disease Center of PLA, The 81st Hospital of PLA, Nanjing, Jiangsu, China; ${ }^{16}$ Tangdu Hospital, Air Force Medical University, Xi'an, \\ Shaanxi, China; ${ }^{17}$ Baoji Central Hospital, Baoji, Shaanxi, China; ${ }^{18}$ The First Affiliated Hospital of Wenzhou Medical University, \\ Wenzhou, Zhejiang, China; ${ }^{19}$ The First Hospital Affiliated to Jilin University, Changchun, Jilin, China; ${ }^{20}$ The First Hospital of \\ Changsha, Changsha, Hunan, China; ${ }^{21}$ The First Affiliated Hospital of Guangxi Medical University, Nanning, Guangxi, China; ${ }^{22}$ The \\ First Hospital of Hebei Medical University, Shijiazhuang, Hebei, China; ${ }^{23}$ Beijing Ditan Hospital, Capital Medical University, Beijing, \\ China; ${ }^{24}$ Ascletis BioScience Co., Ltd., Hangzhou, Zhejiang, China
}

\begin{abstract}
Background and Aims: Genotype (GT) 1 remains the predominant hepatitis c virus (HCV) GT in Chinese patients. Over $80 \%$ of those Chinese patients harbor the interferon-sensitive CC allele of IFNL4rs12979860, which is favorable for interferon-based treatment regimens. This phase III clinical trial aimed to evaluate the efficacy and safety of the ritonavirboosted danoprevir plus pegylated-interferon $\alpha-2 \mathrm{a}$ and ribavirin regimen for 12 weeks in treatment-naïve mainland Chinese patients infected with HCV GT1 without cirrhosis. Methods: One hundred and forty-one treatment-naïve, non-cirrhotic HCV GT1 Chinese patients (age $\geq 18$ years) were enrolled for this single-arm, multicenter, phase III MANASA study (NCT03020082). Patients received a combination of ritonavir-boosted danoprevir $(100 \mathrm{mg} / 100 \mathrm{mg})$ twice a day plus subcutaneous injection of weekly pegylated-interferon $\alpha-2 \mathrm{a}(180 \mu \mathrm{g})$ and oral ribavirin $(1000 / 1200 \mathrm{mg} /$ day body weight $<75 / \geq 75 \mathrm{~kg}$ ) for 12 weeks. The primary end-point was sustained virologic response rate at 12 weeks after the end of treatment. The secondary end-points were safety outcomes, tolerability, virologic response over time and relapse rate. Results: All enrolled patients were HCV GT1-infected, and most among them $(97.9 \%, 123 / 141)$ had the HCV GT1b subtype. Single-nucleotide polymorphism test showed that
\end{abstract}

Keywords: Danoprevir; Hepatitis C virus (HCV); Sustained virological response (SVR); Treatment-naïve.

Abbreviations: $A E$, adverse event; $D A A$, direct-acting antiviral agent; $D N V r$, ritonavir-boosted danoprevir; GT, genotype; $\mathrm{HCV}$, hepatitis $\mathrm{C}$ virus; SAE, serious adverse event; SVR, sustained virological response.

Received: 13 May 2019; Revised: 12 June 2019; Accepted: 30 June 2019

* Correspondence to: Lai Wei, Beijing Key Laboratory of Hepatitis C and Immunotherapy for Liver Diseases, Beijing 100191, China. Tel: +86-1-88326666, Fax: +86-1-68318386, E-mail: weilai@pkuph.edu.cn the majority of patients were of the IFNL4 rs12979860 CC genotype $(87.2 \%, 123 / 141)$. Overall, 140 patients completed the 12 -week treatment, and $97.1 \%(136 / 140)$ patients achieved sustained virologic response at 12 weeks (per protocol population group, 95\% confidence interval: 92.9-99.2\%). Only drug-related serious adverse event occurred. Most of the adverse events were grade 1 and grade 2 alanine aminotransferase elevation or liver dysfunction. One patient discontinued treatment because of severe head injury in a car accident. Conclusions: The triple regimen of ritonavir-boosted danoprevir plus pegylated-interferon $\alpha-2 a$ and ribavirin produced a sustained virologic response rate of $97.1 \%$ after 12 weeks treatment in noncirrhotic HCV GT1-infected Chinese patients, and was safe and well tolerated. Trial Registration ClinicalTrials.gov Identifier: NCT03020082

Citation of this article: Wei $L$, Shang J, Ma $Y, X u X$, Huang $Y$, Guan $Y$, et al. Efficacy and safety of 12-week interferon-based danoprevir regimen in patients with genotype 1 chronic hepatitis C. J Clin Transl Hepatol 2019;7(3):221-225. doi: 10.14218/ JCTH.2019.00018.

\section{Introduction}

Chronic hepatitis C virus (HCV) infection is associated with chronic liver diseases, including cirrhosis and hepatocellular carcinoma. ${ }^{1}$ It is estimated that over 10 million Chinese individuals are chronically infected with $\mathrm{HCV} .{ }^{2}$ The annual report on HCV issued by the China Ministry of Health showed a gradual increase of HCV cases from year $2003(20,000$ cases) to $2012(200,000$ cases $) .^{3}$

Studies have shown that the genotype (GT)1b (56.8\%) subtype of $\mathrm{HCV}$ is the most prevalent subtype in China, ${ }^{4}$ 
exhibiting higher proportion of the IFNL4 rs12979860-CC genotype $(>80 \%) .5,6$ To this end, the use of interferon-based therapy produces favorable outcomes on inducing sustained virological response rates of $40-50 \%{ }^{6}$ However, interferonbased therapy is often of long-term duration, creates significant side effects, and suboptimal sustained virological response (SVR). Recently, the real-world study indicated above revealed that most GT1b patients received long-term treatment ${ }^{5}$ (more than 1 year in $50 \%$ percent of patients) than suggested in the $\mathrm{HCV}$ treatment guidelines. Thus, the availability of new drugs may reduce the use of interferon-based therapy.

Currently, the World Health Organization (commonly known as the ' $\mathrm{WHO}$ ') promotes direct-acting antiviral agents (DAAs) as a standard of therapy to eliminate HCV. However, the lofty price tag is becoming an issue, when compared to the cost of interferon-based therapy in China. Alternatively, ritonavir-boosted danoprevir (DNVr) plus pegylated-interferon $\alpha-2 a$ and ribavirin seems reasonable in terms of affordability and side effects. Moreover, danoprevir is the only licensed DAA that has been developed and marketed by Ascletis in China, with the price tag of USD $\$ 965.9 / 2$ weeks.

The macrocyclic noncovalent inhibitors of the HCV NS3/4A enzyme play a vital role against GTs $1,2,4$ and $6,{ }^{7,8}$ with the advantage of having lessened the side effects profile. Several studies has shown that DNVr plus pegylated-interferon $\alpha$ and ribavirin is safe and well tolerated in both Caucasian (DAUPHINE study) and Asian (DAPSANG study) populations. ${ }^{9,10}$ The fixed-dose combination of danoprevir and ritonavir plus pegylated-interferon $\alpha$ and ribavirin achieved $94.1 \%$ SVR12 rate after a 12-week treatment regimen among treatmentnaïve, noncirrhotic HCV GT1 patients in Taiwan. ${ }^{10}$

Our previous phase II study (MAKALU, NCT03020004) showed a $96 \%$ SVR rate in mainland Chinese patients. In the phase III study described herein, we evaluated the efficacy and safety of DNVr plus pegylated-interferon $\alpha-2 a$ and ribavirin regimens for 12 weeks in treatment-naïve mainland Chinese patients infected with HCV GT1 without cirrhosis.

\section{Methods}

\section{Study design}

This phase III, single-arm, multicenter study (termed 'MANASA') aimed to evaluate the efficacy and safety of DNVr $100 \mathrm{mg} / 100 \mathrm{mg}$ twice a day in combination with the regimen in treatment-naïve noncirrhotic GT1 patients, with a superior design to compare the binomial distribution of SVR12 against pegylated-interferon $\alpha-2 \mathrm{a}$ and ribavirin historical data (SVR24 up to $73.9 \%$ ). The study was conducted in full conformance to the ethical principles of the Declaration of Helsinki (2013), and the institutional review board of all participating institutions approved the study (available upon written request to the Corresponding Author). Written informed consent was obtained from all participants according to local laws and regulations of the particular institutions.

\section{Patients and treatment}

This open-label study enrolled 141 adult patients from 23 medical centers of China from June 2016 to July 2017. Eligibility criteria are recorded in detail in the study registration information (NCT03020082). In short, eligible patients were aged $\geq 18$ years and having chronic HCV GT1 infection, with plasma HCV RNA level $>10,000 \mathrm{IU} / \mathrm{mL}$ (prior to screening
Wei L. et al: Danoprevir regimen efficacy in genotype $1 \mathrm{CHC}$

and treatment). Noncirrhosis was defined as METAVIR score of $<$ F3 during screening, or a FibroScan measurement of $\geq 9.6$ $\mathrm{kPa}$ but $<12.9 \mathrm{kPa}$ on liver biopsy. Patients were excluded if they were suspected of hepatocellular carcinoma, had evidence of hepatitis $B$ virus or human immunodeficiency virus coinfection, or had a history of any other chronic liver disease. All patients received DNVr $100 \mathrm{mg} / 100 \mathrm{mg}$ in combination with subcutaneous injection of pegylated-interferon $\alpha-2 a$ $(180 \mu \mathrm{g} /$ week) and ribavirin ( $500 \mathrm{mg}$ body weight $<75 \mathrm{~kg}$ ) or $600 \mathrm{mg}$ (body weight $\geq 75 \mathrm{~kg}$ ) twice a day orally.

\section{Virological assessments}

Virologic response was defined as HCV RNA below the lower limit of quantitation (15 IU/mL) as detected by the COBAS AmpliPrep/COBAS TaqMan Quantitative HCV Test (version 2.0; Roche, Molecular Diagnostics, Rotkreuz, Switzerland). Rapid virologic response was defined as a virologic response at week 4, and SVR was defined as SVR at week 4, 12 or 24 after the end of treatment. Plasma HCV RNA was quantified at screening and at each visiting time thereafter to assess the virologic response to the treatment. Resistance monitoring was performed in all patients at baseline enrollment by sequence analysis of the complete HCV NS3/NS4A coding region; patients who failed to achieve SVR12 included those with viral breakthrough or relapse (detection of HCV RNA during follow-up in a patient with undetectable HCV RNA at end of treatment).

\section{Safety and efficacy outcome}

The primary efficacy end-point was the proportion of patients achieving SVR12. The secondary end-points were safety outcomes, tolerability, virologic response over time, and relapse rate.

\section{Statistical analysis}

All data were analyzed by SAS 9.2 software. A sample size of 71 was required to provide a power of 0.95 with a two-sided significance level of 0.05 to demonstrate the lower boundary of SVR12 of DNVr plus pegylated-interferon $\alpha-2 a$ and ribavirin superiority to the pegylated-interferon $\alpha-2 a$ and ribavirin regimen. A $p$-value $<0.05$ was considered statistically significant. Our primary meta-analysis (data not shown) indicated that the average SVR24 rate in mainland Chinese HCV patients infected with GT1 for the pegylated-interferon $\alpha-2 a$ and ribavirin regimen was $73.9 \%$, and the expected SVR12 rate of the DNVr plus pegylated-interferon $\alpha-2 a$ and ribavirin regimen was $90 \%$.

\section{Results}

\section{Study population}

A total of 141 patients received $\geq 1$ dose of the DNVr plus pegylated-interferon $\alpha-2 \mathrm{a}$ and ribavirin regimen, among them 138/141 (87.8\%) had IFNL4 rs12979860 (a single nucleotide polymorphism on chromosome 19, rs12979860) CC GT, which is favorable for interferon-based treatment, and three patients were infected with HCV GT1a (3/141, 2.1\%) (Table 1). In total, $140 / 141(97.1 \%)$ patients completed the treatment. One (1.4\%) patient discontinued treatment due to treatment-unrelated serious adverse events (SAEs). 
Wei L. et al: Danoprevir regimen efficacy in genotype $1 \mathrm{CHC}$

Table 1. Patient demographics and disease characteristics

\begin{tabular}{ll}
\hline Variables & $n=141$ \\
\hline Age in years, mean (range) & $42.1(19.0 \sim 72.2)$ \\
Gender as m/f, $\boldsymbol{n}$ & $71 / 70$ \\
BMI in kg/ $\mathbf{2}^{\mathbf{2}}$, mean (range) & $23.0(18.1 \sim 29.7)$ \\
Nationality of Han/other, $\boldsymbol{n}$ & $126 / 49$ \\
Baseline HCV RNA in log $\mathbf{1 0}$ & $6.04(2.91 \sim 7.08)$ \\
IU/mL, median (range) & \\
Baseline HCV RNA in & \\
IU/mL, $\boldsymbol{n}$ (\%) & $33(23.4)$ \\
$\quad<400,000$ & $14(9.9)$ \\
$400,000-800,000$ & $94(66.7)$ \\
$\geq 800$ 000 & \\
IFNL4 rs12979860, $\boldsymbol{n}$ (\%) & $123(87.2)$ \\
CC & $18(12.8)$ \\
non-CC & \\
HCV genotype, $\boldsymbol{n}$ (\%) & $3(2.1)$ \\
1a & $138(97.9)$ \\
1b & $6.1(2.7 \sim 12.2)$ \\
FibroScan (kPa), mean (range) & \\
\hline
\end{tabular}

Abbreviations: BMI, body mass index; $\mathrm{HCV}$, hepatitis $\mathrm{C}$ virus; $\mathrm{kPa}$, kilopascals.

\section{Virologic response over time}

At week 1, the HCV RNA levels of all individual patients declined rapidly after treatment initiation with DNVr plus pegylatedinterferon $\alpha-2 a$ and ribavirin (Fig. 1). Among 141 patients enrolled, 140 patients completed the 12-week therapy and one patient withdrew due to treatment-unrelated SAEs. A total of 136 of 141 patients (intention-to-treat group, $96.5 \%$, [95\% confidence interval (CI): 91.9-98.8\%]) achieved SVR12 (Fig. 2), with the lower boundary of the $95 \%$ CI for the DNVr plus pegylated-interferon $\alpha-2 a$ and ribavirin regimen higher than the SVR12 of $73.9 \%$ in another pegylated-interferon $\alpha-2 \mathrm{a}$ and ribavirin regimen study (based on historical results of the pegylated-interferon $\alpha-2 a$ and ribavirin regimen in Chinese chronic hepatitis $C$ patients, $p<0.001$ ).

Overall, among the 140 patients who completed the treatment, $118 / 122(96.7 \%)$ with the IFNL4 rs12979860 CC genotype and $18 / 18(100.0 \%)$ with the CT genotype achieved SVR12. A total of 139 [98.6\%, (95\% CI: 96.1-100\%)] patients achieved virologic responses by the end of the treatment ( $\mathrm{HCV}$ RNA $<15 \mathrm{IU} / \mathrm{mL}$ ). One patient discontinued the treatment on day 10 due to car accident.

\section{Resistance}

No virologic breakthrough occurred during the study. Four cases $(2.9 \%, 4 / 140)$ experienced relapse, among which three were detected at week 4 after end of treatment and one at week 12 after end of treatment. Baseline gene sequencing of amino acid polymorphisms at NS3 positions revealed that three relapsed patients exhibited NS3-D168A polymorphisms, while one patient had NS3-R155K polymorphisms.

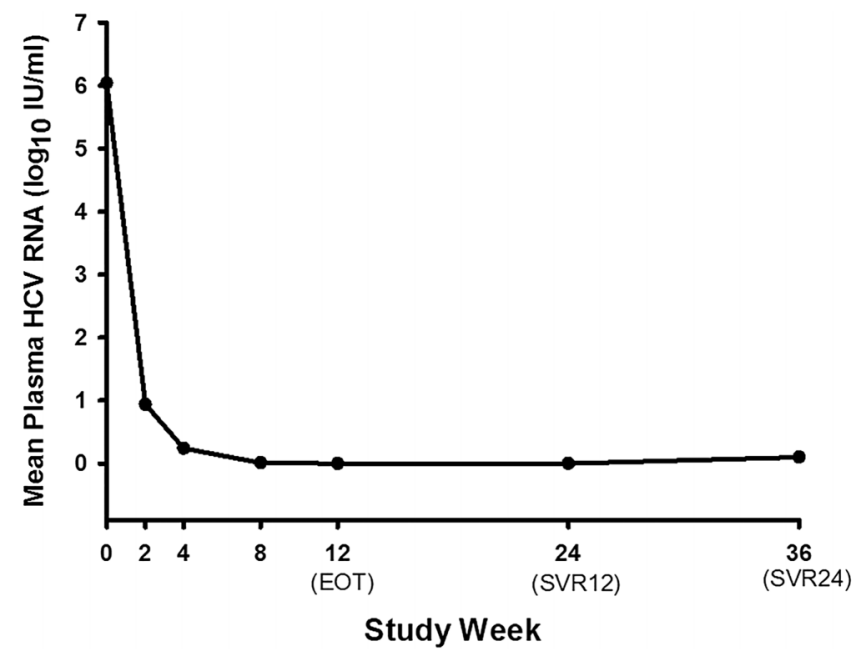

Fig. 1. Mean plasma HCV RNA change during the treatment of all the patients who completed the treatment. The HCV RNA levels of all individual patients declined rapidly after initiation of treatment with DNVr plus pegylatedinterferon $\alpha-2$ a and ribavirin on week 1 . Plasma HCV RNA was quantified at screening and at each visit thereafter to assess the virologic response to treatment. Plasma HCV RNA was recorded as $1 \mathrm{IU} / \mathrm{mL}$ (equal to $1 \log 10 \mathrm{IU} / \mathrm{mL}$ ) if HCV RNA $<15 \mathrm{IU} / \mathrm{mL}$ (lower limit of quantitation) or target not detected.

Abbreviations: HCV, hepatitis C virus; DNVr, ritonavir-boosted danoprevir; EOT, end of treatment; SVR12, sustained virologic response at 12 weeks after treatment; SVR24, sustained virologic response at 24 weeks after treatment.

\section{Safety}

The safety profiles of DNVr plus pegylated-interferon $\alpha-2 a$ and ribavirin was comparable to that of pegylated-interferon $\alpha-2 a$ and ribavirin therapy. At least one adverse event (AE) was reported in all patients. Most AEs were of mild to moderate in severity. AEs that were observed in more than $10 \%$ of the patients during treatment and follow-up are shown in Table 2. Anemia, fever, fatigue, leukopenia, neutropenia and

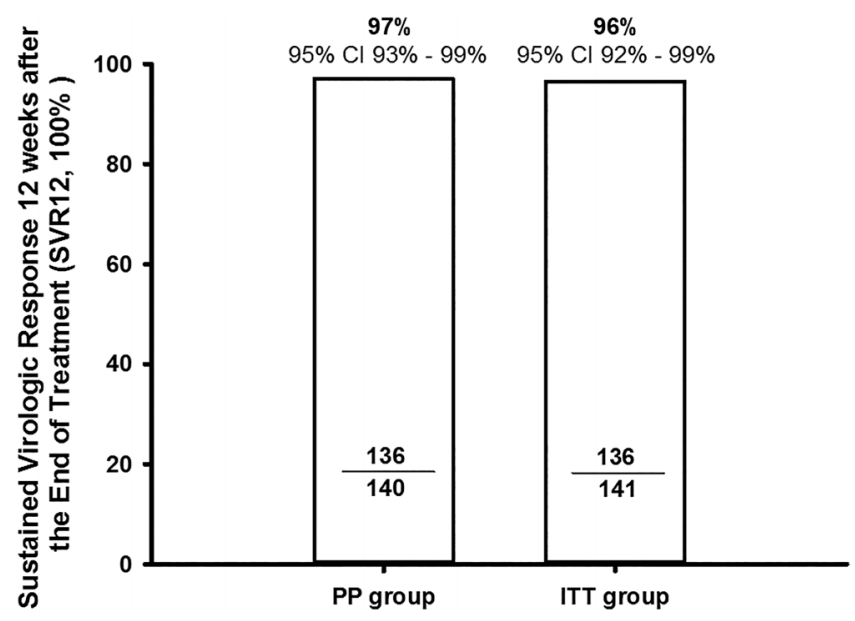

Fig. 2. SVR 12 weeks after the end of treatment (SVR12) in PP group $(n=140)$ and ITT group $(n=141)$. One patient discontinued treatment due to car accident.

Abbreviations: SVR, sustained virologic response; PP, per protocol population; ITT, intention-to-treat. 
Table 2. Adverse events and laboratory abnormalities

\begin{tabular}{|c|c|}
\hline Event or abnormality & $n(\%)$ \\
\hline Any adverse events & $141(100.0)$ \\
\hline Severe adverse events* & $5(3.5)$ \\
\hline Adverse events leading to discontinuation" & $1(0.0)$ \\
\hline \multicolumn{2}{|l|}{ Adverse events in $\geq 10 \%$ of patients } \\
\hline Anemia & $76(53.9)$ \\
\hline Fever & $53(37.6)$ \\
\hline Fatigue & $52(36.9)$ \\
\hline Headache & $31(22.0)$ \\
\hline Dizziness & $28(19.9)$ \\
\hline Anorexia & $26(18.4)$ \\
\hline Diarrhea & $25(17.7)$ \\
\hline Flu-like symptoms & $25(17.7)$ \\
\hline Hypertriglyceridemia & $21(14.9)$ \\
\hline Erythra & $17(12.1)$ \\
\hline Urinary tract infection & $16(11.34)$ \\
\hline Nausea & $13(19.5)$ \\
\hline \multicolumn{2}{|c|}{ Laboratory abnormalities in $\geq 10 \%$ of patients } \\
\hline Leukopenia & $118(83.7)$ \\
\hline Neutropenia & $103(73.1)$ \\
\hline Thrombocytopenia & $51(36.2)$ \\
\hline Lymphocytosis & $26(14.2)$ \\
\hline High ALT & $24(17.0)$ \\
\hline Hypochromia & $22(16.3)$ \\
\hline High AST & $19(13.5)$ \\
\hline Erythropenia & $19(13.5)$ \\
\hline
\end{tabular}

* One severe adverse event of the pegylated-interferon and ritonavir regimen, but not the ritonavir-boosted danoprevir regimen, and three severe adverse events not related to treatment regimen.

\# One car crash death was not related with treatment and the patient was excluded from the study.

Abbreviations: ALT, alanine aminotransferase; AST, aspartate aminotransferase.

thrombocytopenia occurred in more than $30 \%$ patients, which was most likely to be consistent with AEs of ribavirin and pegylated-interferon treatment. Abnormal liver function related to pegylated-interferon and ribavirin were mainly classified into grades 1 and 2 (Table 3 ).

Six SAEs occurred in five patients (Table 4), which included recurrence of tuberculosis, neutropenia, ventricular tachyarrhythmia, transient ischemic attack, and digital fibrokeratoma. All five patients continued treatment despite the SAEs. A 50-year-old patient withdrew from the study because of severe head injury in a car accident. Only neutropenia was considered to be related with pegylated-interferon, ribavirin, danoprevir, or ritonavir.

\section{Discussion}

In this phase III study, 141 treatment-naïve mainland Chinese patients infected with HCV GT1 without cirrhosis was evaluated for 12-weeks to determine the efficacy and safety of DNVr plus pegylated-interferon $\alpha-2 a$ and ribavirin
Wei L. et al: Danoprevir regimen efficacy in genotype $1 \mathrm{CHC}$

Table 3. Abnormal liver function test results

\begin{tabular}{llll}
\hline Feature & $\begin{array}{l}\text { Grade 1, } \\
n(\%)\end{array}$ & $\begin{array}{l}\text { Grade 2, } \\
n(\%)\end{array}$ & $\begin{array}{l}\text { Grades 3 and } \\
4, n(\%)\end{array}$ \\
\hline High ALT & $10(7.1)$ & $3(2.1)$ & 0 \\
High AST & $10(7.1)$ & $1(0.7)$ & 0 \\
High TBIL & $13(9.2)$ & 0 & 0 \\
High DBIL & $3(2.1)$ & 0 & 0 \\
\hline
\end{tabular}

Abbreviations: ALT, alanine aminotransferase; AST, aspartate aminotransferase; TBIL, total bilirubin; DBIL, direct bilirubin.

regimens. This phase III treatment regimen produced $97 \%$ SVR12, and thus was safe and effective. Overall, this finding matches that of phase II (96\% SVR12) and the DAPSANG study (94.1\% SVR12). In the sofosbuvir registration study in China, sofosbuvir plus pegylated-interferon $\alpha-2 a$ and ribavirin 12-week therapy showed 94\% SVR12, and in the simeprevir registration study in China, simeprevir plus pegylated-interferon $\alpha-2 a$ and ribavirin 24/48-week therapy showed $91 \%$ SVR12. Compared with these two other DAA plus pegylatedinterferon $\alpha-2 a$ and ribavirin regimens, danoprevir shows better efficacy with a short treatment duration. In addition, it also showed a better efficacy and shorter treatment duration than the pegylated-interferon $\alpha-2 a$ and ribavirin regimen [first generation protease inhibitor plus pegylated-interferon $\alpha-2 a$ and ribavirin regimens $(67-75 \%)^{11}$ ] and other DAA plus pegylated-interferon $\alpha-2 a$ and ribavirin regimens (sofosbuvir plus pegylated-interferon $\alpha-2 a$ and ribavirin, simeprevir plus pegylated-interferon $\alpha-2 a$ and ribavirin, daclatasvir plus pegylatedinterferon $\alpha-2 a$ and ribavirin). ${ }^{9,12-14}$

According to the literature, virologic breakthrough and relapse are concerns during the treatment. Interestingly, in our phase II and III trials, no virologic breakthrough occurred but four patients with previously documented baseline NS3D168 or NS3-R155 polymorphisms experienced relapse at weeks 4 and 12 after the end of the treatment. Notably, patients enrolled in our phase II and III studies of the DNVr plus pegylated-interferon $\alpha-2 a$ and ribavirin regimen without baseline resistant-associated substitutions screening achieved $>95 \%$ SVR12, suggesting that there was no need

Table 4. Summary of severe adverse events during and after the treatment

\begin{tabular}{|c|c|c|}
\hline Events & $n(\%)$ & $\begin{array}{l}\text { Relation to DNVr, } \\
\text { PEG-IFN, RTV and RBV }\end{array}$ \\
\hline Neutropenia & $1(0.7)$ & PEG-IFN, RBV, RTV, DNVr \\
\hline Tuberculosis & $1(0.7)$ & $\mathrm{N} / \mathrm{A}$ \\
\hline $\begin{array}{l}\text { Ventricular } \\
\text { tachyarrhythmia }\end{array}$ & $1(0.7)$ & PEG-IFN, RBV \\
\hline $\begin{array}{l}\text { Transient } \\
\text { ischemic attack }\end{array}$ & $1(0.7)$ & N/A \\
\hline $\begin{array}{l}\text { Acquired digital } \\
\text { fibrokeratoma }\end{array}$ & $1(0.7)$ & $\mathrm{N} / \mathrm{A}$ \\
\hline $\begin{array}{l}\text { Craniocerebral } \\
\text { injury }\end{array}$ & $1(0.7)$ & Possibly not \\
\hline
\end{tabular}

Abbreviations: PEG-IFN, pegylated-interferon; DNVr, ritonavir-boosted danoprevir; RBV, ribavirin; RTV, ritonavir; N/A, not applicable. 
for baseline resistant-associated substitutions screening before using this regimen.

The DNVr plus pegylated-interferon $\alpha-2 a$ and ribavirin regimen was safe and well tolerated. Most AEs were related to pegylated-interferon $\alpha-2 a$ and ribavirin and only grade $1-2$ liver dysfunctions were observed (Table 3). The most frequently reported AEs (in $>30 \%$ ) were anemia, fever, fatigue, leukopenia, neutropenia and thrombocytopenia, of which only neutropenia was considered to be caused by danoprevir, while others were consistent with AEs of ribavirin and pegylated-interferon treatment. One patient withdrew from the study because of brain injury (and ultimate death) following a serious car accident during the treatment. No patient discontinued the study because of AEs.

Evidence suggests that the all-oral DAA regimen from foreign pharmaceutical companies that are currently about to be launched may cost USD $\$ 8376$ to $\$ 17,092$ for each course, ${ }^{15}$ but the per capita disposable income of Chinese residents in 2017 was only USD $\$ 3763.6 .{ }^{16}$ In addition, a medical insurance scheme for DAAs therapy is only valid in three provinces of China. Thus, most patients are still unable to afford the imported all-oral DAA regimens. Danoprevir, being the first DAA developed by a Chinese company (Ascletis) and having the more affordable price tag of USD $\$ 965.9 / 2$ weeks, seems affordable, with the cost of each $1 \%$ increase in SVR of USD $\$ 77.6$ (danoprevir plus pegylated-interferon $\alpha-2 a$ and ribavirin regimen), which is lower than that of foreign made all-oral DAA regimens (USD $\$ 85.8$ to $\$ 179.9$ for each $1 \%$ increase in SVR) and pegylated-interferon $\alpha-2$ a and ribavirin regimens (USD $\$ 89.3$ to $\$ 133$ for each $1 \%$ increase in SVR). The overall treatment cost may become lower, if the cost of pegylated-interferon $\alpha-2 a$ and ribavirin (USD $\$ 288.4 / 2$ weeks) gets reimbursed by a medical insurance scheme in all provinces. ${ }^{15}$

The limitation of our study includes small sample size restricted to the inclusion and exclusion criteria, as defined by clinical trial protocols. Another concern was the weekly injection, which leads to poorer adherence in real-world practices. Finally, real-world studies on large-scale should be initiated for additional information.

\section{Conclusions}

In all, the treatment goal for hepatitis $C$ is not only to clear $\mathrm{HCV}$ but also to improve disease outcome and long-term prognosis. The DAA plus pegylated-interferon $\alpha-2 a$ and ribavirin regimens, which produce dual effect on immunomodulation and antivirus activity, could improve the efficacy and shorten the chronic hepatitis $\mathrm{C}$ virus treatment course, and thereby reduce the occurrence of cirrhosis, hepatocellular carcinoma, and HCV-related death.

\section{Acknowledgments}

Ascletis Pharmaceuticals Co., Ltd. provided financial support for this study (MANASA).

\section{Conflict of interest}

Lai Wei is a former consultant for AbbVie, Abbott, BMS, Gilead Sciences, Johnson \& Johnson, MSD, Novartis and Roche, speaker for Abbott, BMS, Gilead Sciences, MSD, Novartis, Roche and Ascletis, and received grant support from BMS and Roche. Lan Zhang, Huoling Tang, Jinzi J. Wu were employees of Ascletis Pharmaceuticals Company. The others have no conflict of interests related to this publication.

\section{Author contributions}

Study concept and design (LW and JJW), performed the study, recruited patients and collected specimens (LW, JS, YM, XX, $Y H, Y G, Z D, W Z, Z G, M Z, J L, J J, Y Y, X W, M W, Z J, B N, Y C, Y Q$, $J \mathrm{D}, \mathrm{JJ}, \mathrm{LT}$ and $Y X)$, and drafted the manuscript (LW).

\section{References}

[1] Guidelines for the screening care and treatment of persons with chronic hepatitis C infection: Updated version. Geneva: World Health Organization, 2016.

[2] Global prevalence and genotype distribution of hepatitis $C$ virus infection in 2015: a modelling study. Lancet Gastroenterol Hepatol 2017;2:161-176. doi: 10.1016/S2468-1253(16)30181-9.

[3] Duan Z, Jia JD, Hou J, Lou L, Tobias H, Xu XY, et al. Current challenges and the management of chronic hepatitis $C$ in mainland China. J Clin Gastroenterol 2014;48:679-686. doi: 10.1097/MCG.0000000000000109.

[4] Rao H, Wei L, Lopez-Talavera JC, Shang J, Chen H, Li J, et al. Distribution and clinical correlates of viral and host genotypes in Chinese patients with chronic hepatitis C virus infection. J Gastroenterol Hepatol 2014;29:545-553. doi: 10.1111/jgh.12398.

[5] Rao HY, Li H, Chen H, Shang J, Xie Q, Gao ZL, et al. Real-world treatment patterns and clinical outcomes of HCV treatment-naive patients in China: an interim analysis from the CCgenos study. J Gastroenterol Hepatol 2017;32: 244-252. doi: 10.1111/jgh.13467.

[6] Manns MP, McHutchison JG, Gordon SC, Rustgi VK, Shiffman M, Reindollar R, et $a$. Peginterferon alfa- $2 b$ plus ribavirin compared with interferon alfa- $2 b$ plus ribavirin for initial treatment of chronic hepatitis $\mathrm{C}$ : a randomised trial. Lancet 2001;358:958-965. doi: 10.1016/s0140-6736(01)06102-5.

[7] Jiang Y, Andrews SW, Condroski KR, Buckman B, Serebryany V, Wenglowsky S, et al. Discovery of danoprevir (ITMN-191/R7227), a highly selective and potent inhibitor of hepatitis C virus (HCV) NS3/4A protease. J Med Chem 2014;57:1753-1769. doi: 10.1021/jm400164C.

[8] Gottwein JM, Scheel TK, Jensen TB, Ghanem L, Bukh J. Differential efficacy of protease inhibitors against HCV genotypes $2 a, 3 a, 5 a$, and $6 a$ NS3/4A protease recombinant viruses. Gastroenterology 2011;141:1067-1079. doi: 10.1053/j.gastro.2011.06.004

[9] Everson G, Cooper C, Hézode C, Shiffman ML, Yoshida E, Beltran-Jaramillo T, et al. DAUPHINE: a randomized phase II study of danoprevir/ritonavir plus peginterferon alpha-2a/ribavirin in HCV genotypes 1 or 4 . Liver Int $2015 ; 35$ : 108-119. doi: 10.1111/liv.12471.

[10] Kao JH, Tung SY, Lee Y, Thongsawat S, Tanwandee T, Sheen IS, et al. Ritonavir-boosted danoprevir plus peginterferon alfa-2a and ribavirin in Asian chronic hepatitis C patients with or without cirrhosis. J Gastroenterol Hepatol 2016;31:1757-1765. doi: 10.1111/jgh.13374.

[11] Wei L, Lok AS. Impact of new hepatitis $C$ treatments in different regions of the world. Gastroenterology 2014;146:1145-1150.e4. doi: 10.1053/j. gastro.2014.03.008

[12] Lawitz E, Mangia A, Wyles D, Rodriguez-Torres M, Hassanein T, Gordon SC, et al. Sofosbuvir for previously untreated chronic hepatitis $C$ infection. N Engl J Med 2013;368:1878-1887. doi: 10.1056/NEJMoa1214853.

[13] Wei L, Han T, Yang D, Heo J, Shang J, Cheng J, et al. Simeprevir plus peginterferon/ribavirin for HCV genotype 1-infected treatment-naïve patients in China and South Korea. J Gastroenterol Hepatol 2016;31:912-920. doi: 10. $1111 /$ jgh. 13288

[14] Hézode C, Hirschfield GM, Ghesquiere W, Sievert W, Rodriguez-Torres M, Shafran SD, et al. Daclatasvir plus peginterferon alfa and ribavirin for treatment-naive chronic hepatitis $C$ genotype 1 or 4 infection: a randomised study. Gut 2015;64:948-956. doi: 10.1136/gutjnl-2014-307498.

[15] Hu SL, Lu J. A comparative cost-effectiveness analysis on direct-acting antiHCV agents. China Health Insurance 2018;8:53-58.

[16] National Bureau of Statistics of China. Statistical Communiqué of the People's Republic of China on the 2017 National Economic and Social Development. Available from: http://www.stats.gov.cn/english/PressRelease/201802/t20180228_1585666.html. Accessed February 28, 2018. 